\title{
Changes in skin potential level during sleep in psychiatric patients
}

\author{
RICHARD PERKINS* \\ M.B., B.S., M.R.C.Psych., D.D.M. \\ MARTYN BAKER $\dagger$ \\ B.A.
}

\begin{abstract}
*Watford General Hospital and $\dagger$ Academic Department of Psychiatry, Middlesex Hospital Medical School, London $W 1 N 8 A A$
\end{abstract}

\begin{abstract}
Summary
Skin potential levels and EEG changes were recorded in eight psychiatric patients during three nights of sleep. In a balanced design each patient took amylobarbitone sodium $200 \mathrm{mg}$, chlordiazepoxide $30 \mathrm{mg}$ and placebo in turn. Skin potential did not distinguish between wakefulness and sleep as measured by the EEG nor did it clearly identify individual sleep stages. However, significant differences in skin potential were found between Awake and Stage I, between Awake and REM sleep and between REM sleep and Stage III/IV. The level was lowest during REM sleep but approached that of wakefulness during slow-wave sleep. These findings are discussed in terms of changes in arousal threshold during sleep. A cautious comment is made on the possible effects of psychiatric diagnoses and drugs.
\end{abstract}

\section{Introduction}

Electrodermal activity in human subjects has been studied in two separate ways. It has either been measured indirectly as skin resistance (or conductance) or directly as skin potential. In both cases absolute levels and spontaneously occurring fluctuations have been measured. Electrodermal activity has long been regarded as a useful measure of arousal in the waking state. With regard to skin resistance the number of spontaneously occurring fluctuations has been found to be especially reliable in this respect (Johnson, 1963; Lader and Wing, 1964). Leiderman and Shapiro (1964) showed that skin potential level was a useful measure of arousal in the waking state.

There is also a possibility that electrodermal activity might differentiate between varying levels of arousal during the sleeping state. Several studies of electrodermal activity during sleep in normal subjects have been done and attempts have been made to correlate changes in electrodermal activity with the various EEG stages of sleep. Johnson and Lubin (1966) confirmed earlier studies in showing that spontaneous fluctuations in skin resistance and skin potential occurred during sleep and that these were increased in slow-wave sleep (Stages III and IV) and greatly reduced during REM sleep. Koumans, Tursky and Solomon (1968) recorded all night skin potential and skin resistance levels and rapid electrodermal fluctuations during sleep. They found that skin resistance and skin potential levels could differentiate between wakefulness and sleep but could not clearly differentiate between individual EEG sleep stages. Spontaneous rapid fluctuations in both skin resistance and skin potential were recorded throughout sleep but again no pattern was found that could clearly identify individual sleep stages. This frustrated their hope of being able to replace EEG recording with skin potential recording in order to monitor sleep. However, they did find an increase in the number of fluctuations occurring in slow-wave sleep and a decrease during REM sleep. The latter in fact occurred a few minutes before the onset of dream sleep and they suggested that this measure could be used as a cue to wake subjects in sleep deprivation studies.

The present study evolved in the following way. A hypnotic drug trial was being conducted utilizing allnight EEG recordings (Perkins and Hinton, 1974). This provided a good opportunity to record skin potential levels throughout sleep in psychiatric patients who were taking two different night sedatives. The aims were to see whether we could confirm the finding cited above that skin potential level could differentiate between wakefulness and sleep, in which case it might prove a useful measurement of sleep in hypnotic drug trials and furthermore to make our own comparisons between changes in skin potential level and individual sleep stages. It must be noted that we were only studying skin potential level and not spontaneous fluctuations for reasons explained below.

\section{Methods}

Subjects

Eight in-patients (one man and seven women) at St Luke's Woodside Hospital between the ages of 20 and 60 were studied. Six of the subjects were suffering from anxiety states and two from depressive 
illnesses. They were taking part in a double-blind balanced crossover design trial between amylobarbitone sodium and chlordiazepoxide. Patients slept in a quiet room adjacent to the recording room. The night sedative was taken at 11.00 p.m. and sleep was monitored from 11.00 p.m. until 7.00 a.m. They received no other medication or ECT during the day.

\section{EEG recordings}

EEG recordings were made using five channels of a polygraph recorder (three EEG and two REM channels). Paper speed was $10 \mathrm{~mm} / \mathrm{sec}$. The polygraph was switched on for $1-\mathrm{min}$ periods at 10 -min intervals and each 1-min epoch was allotted one of the sleep stages described by Rechtschaffen and Kales (1968). However their internationally agreed criteria could not be followed exactly because these had been devised using epochs of shorter duration which were part of a continuous rather than intermittent tracing. Also electromyographic recordings were not done in the present investigation.

\section{Skin potential recordings}

A skin potential tracing was recorded on one channel of the polygraph. Silver-silver chloride sponge electrodes with low bias and drift developed by O'Conell and Tursky (1960) were used. The electrode core was surrounded by a pad of cotton wool soaked in a $0.5 \% \mathrm{KCl}$ electrolyte solution. One electrode was placed over the intact skin of the hypothenar eminence of the left hand and the other was placed over an abraded site on the left forearm, 6 in from the wrist. Skin potential was recorded directly from the electrodes through the high input impedance of the polygraph pre-amplifier. The sensitivity, worked at $20 \mathrm{mV} / \mathrm{cm}$, was not sufficient to record small fluctuations and therefore skin potential levels only were recorded. The level taken was that occurring at the end of each 1-min EEG epoch.

\section{Results}

For the purpose of this study we looked only at the results for each patient under the following three conditions: amylobarbitone sodium $200 \mathrm{mg}$; chlordiazepoxide $30 \mathrm{mg}$ and placebo, since the ratio between the two drugs at this dosage is approximately the same as that found to give equivalent effects in relieving anxiety (Lader and Wing, 1965). The mean values of the first ten recordings of skin potential level at each sleep stage for each condition were analysed for each patient. The distribution of the data was not normal, so a log transformation was used and the data were subjected to a split-plot analysis of variance. The greatest variance for skin potential was found between subjects $(F=17 \cdot 853$, d.f. $=7, P \leqslant 0.001$ ), followed by that between stages $(\mathrm{F}=9 \cdot 458$, d.f. $=4, P \leqslant 0.001)$. Significant variance was not found between drugs.

Of the two-way interactions, drugs $\times$ subjects showed significant variance $(\mathrm{F}=10 \cdot 202$, d.f. $=13, \stackrel{-}{\Rightarrow}$ $P \leqslant 0.001)$ as did stages $\times$ subjects $(F=2.232$, d.f. $=8, P \leqslant 0.025$ ).

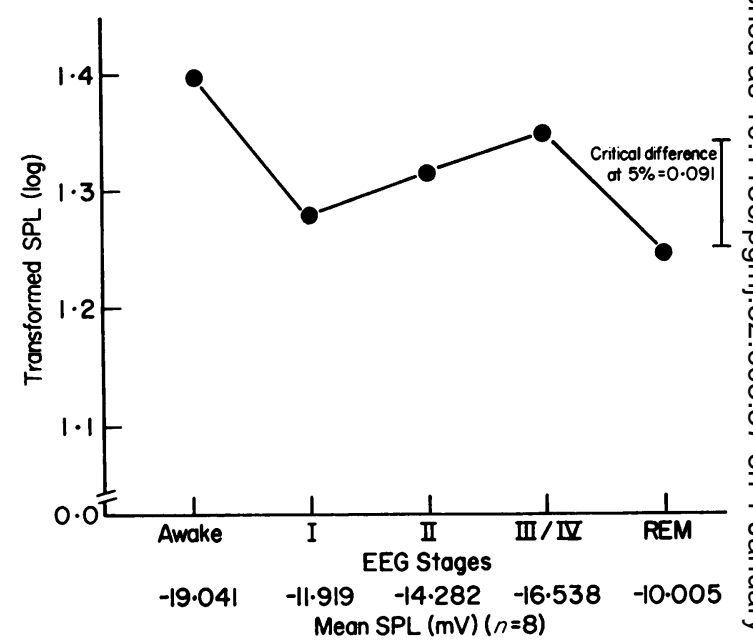

FIG. 1. Mean skin potential level at five EEG stages.

Figure 1 shows mean skin potential levels at five EEG stages. The S-method (Scheffé, 1953) was used to evaluate the comparison between means. The only differences that reached significance were $\frac{\varnothing}{\varnothing}$ between Awake and Stage I $(t=4.340, P \leqslant 0.01)$, between Awake and REM sleep $(t=5.585, P \leqslant 0.01) \overrightarrow{\overrightarrow{0}}$ and between REM sleep and Stage III/IV $(t=3 \cdot 639$, 3 $P \leqslant 0.025$ ). The lowest (least negative) point is reached during REM sleep although it is not significantly lower than that reached at Stages I and II. At Stage III/IV the level is the highest found during sleep.

With regard to subject differences, as there were only two depressed subjects it was not valid to do a $\frac{\text { 의 }}{3}$ statistical comparison between the anxious and the depressed patients. However out of interest the mean levels for these two 'groups' at each sleep stage $\frac{D}{0}$ are plotted separately in Fig. 2. Skin potential is also plotted against drugs for the two diagnostic cate- $N$ gories (Fig. 3). A cautious comment is made below $N$ on these two graphs.

\section{Discussion}

The results showed that skin potential level could not be used to distinguish between sleep and wake- $\mathbb{\Phi}$ fulness. This is contrary to the finding of Koumans ? et al. (1968). It frustrated our hope that in future $\frac{0}{0}$ hypnotic drug trials skin potential level might be 


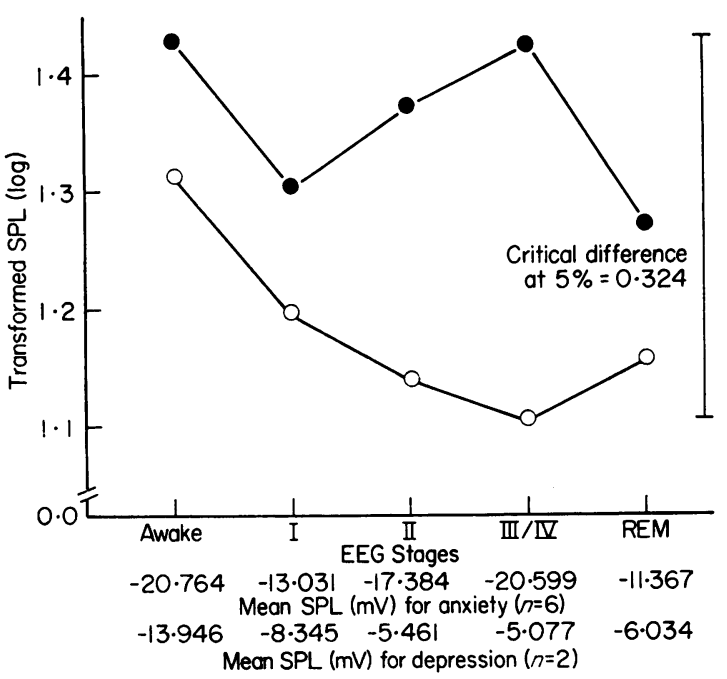

FIG. 2. Mean skin potential level at five EEG stages for anxious, $O$, and depressed, $\bigcirc$, subjects.

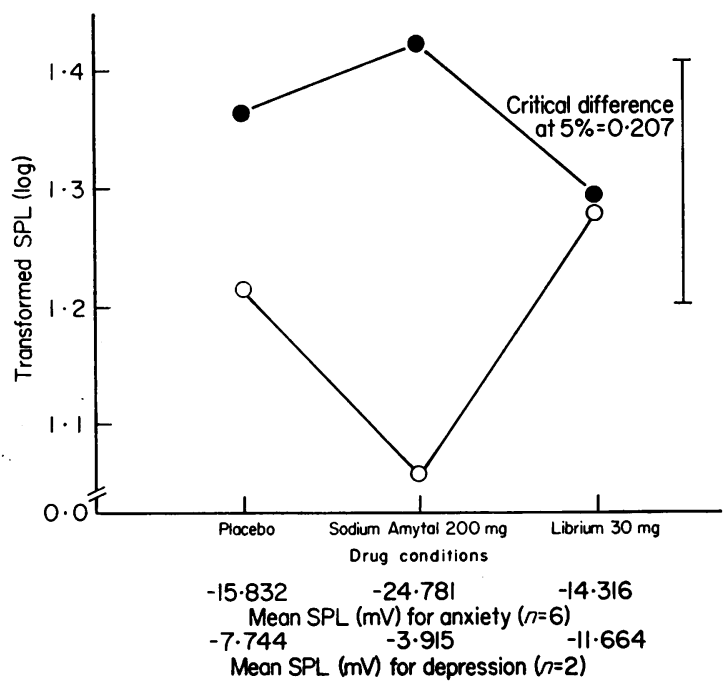

FIG. 3. Mean skin potential level under three drug conditions for anxious, $\bigcirc$, and depressed, $\bigcirc$, subjects.

used on its own as a way of recording the total time spent asleep. Similarly it was not possible to identify each individual sleep stage by changes in skin potential level. However as skin potential did distinguish between Awake and Stage I it might be useful as an indicator of initial transition into sleep.

The finding that skin potential level significantly decreased during REM sleep but returned during slow-wave sleep (Stage III/IV) to a level approaching that found during wakefulness would seem to be related to the findings of Johnson and Lubin (1966) and Koumans et al. (1968) with regard to changes in the number of electrodermal fluctuations. Both these groups of authors attempt to explain these changes in terms of the influence of a rhombocephalic inhibitory centre. At a different level of explanation we would like to interpret our findings in terms of changes in arousal.

If one is considering skin potential level as a measure of level of arousal during sleep it might seem paradoxical that the level increases during slowwave sleep, which is often considered as the deepest part of sleep. Also as dream sleep would appear to consist of more mental activity one might have expected this to be a state of higher arousal. Studies of single neurone units in animals have shown that in certain areas of the brain there is a reduction in activity during the transition from wakefulness to slow-wave sleep and then an increase in activity during desynchronized sleep to a level as high or higher than that found during wakefulness (McCarley and Hobson, 1971). However, the dream stage of sleep has rightly been called paradoxical and one of the paradoxical findings is that although the EEG recording looks like an 'aroused recording', and indeed a period of wakefulness or Stage I sleep often follows a period of dream sleep, animal studies have shown that the actual arousal threshold to external stimulation is higher during activated sleep than during slow-wave sleep (Dillon and Webb, 1965). It may be that our findings of changes in skin potential levels are indicative of changing arousal thresholds.

The mechanism of skin potential level as a measure of arousal is less clear than that of skin conductance. The latter depends primarily on sweat gland activity (Lader and Montagu, 1962) but Venables and Martin (1967) showed that when sweating is abolished pharmacologically there still exists a skin potential level which is of less magnitude (i.e. less negativity). Furthermore, Christie and Venables (1971a) showed that a similar level of potential resulted when sweating was reduced by relaxing subjects over a short period of time. They called this 'basal' skin potential level and went on to produce evidence that this is a diffusion potential across a semi-permeable membrane and therefore related to electrolyte concentration (potassium in this case as the external electrolyte is $\mathrm{KCl}$ ) on either side of the membrane (Christie and Venables, 1971b, c). They commented that this presented problems if skin potential level was required as an index of arousal in low arousal states but that it might prove useful in monitoring electrolyte changes. The mean value found for basal skin potential level by Christie and Venables was $-18 \mathrm{mV}$. The mean value we found for wakefulness was $-19.04 \mathrm{mV}$. All the values for the other stages were below this which suggests that throughout sleep 
we were in the so-called 'basal' skin potential range. It is therefore possible, if Christie and Venables are right in their theory, that we were monitoring changes in electrolyte balance throughout sleep rather than changes in autonomic activity.

From a different standpoint our finding that REM sleep and Stage III/IV were the most clearly distinct areas of sleep with regard to skin potential level falls in line with the idea put forward by Hartmann (1973) that these two areas are indeed the most physiologically distinct and serve totally different functions for the organism.

As has already been stated, because there were only two depressed subjects little weight can be attached to differences found between the anxious and depressed subjects. Nevertheless, Fig. 2 shows an interesting effect. Not only is the overall level of skin potential lower in the depressed subjects but also an entirely different pattern is displayed throughout the sleep stages. Thus, the anxiety curve follows the general curve shown in Fig. 1 but the depressed curve shows a reversed trend. This is most marked at Stage III/IV where in the depressed subjects the lowest level of skin potential is recorded. The lower levels for the depressed subjects would fit in with the concept of reduced physiological function during depression. Lader and Wing (1969) and Noble and Lader $(1971,1972)$ demonstrated reduced electrodermal activity during the waking state as measured by skin conductance in certain forms of depression. All that can be said at this stage is that the present paper represents only a preliminary study and that further investigation is probably justified in order to examine possible differences in this respect between depressed and anxious subjects. (If the trend towards lower levels of skin potential during sleep for depressed subjects were confirmed one might be tempted to interpret it either in terms of altered levels of arousal or, if one favours Venables and Christie's theory of 'basal' skin potential, in terms of altered electrolyte balance. Electrolyte changes have been reported in depression. Those concerning sodium have been the most consistent but Shaw and Coppen (1966) also found body potassium and intracellular potassium were low during depression but did not change with recovery.)

Finally, Fig. 3 suggests that there may be a drug effect superimposed upon the possible between subject differences. Amylobarbitone sodium appears to exaggerate the difference between the depressed and anxious subjects whereas chlordiazepoxide eliminates it. However, as before, because of the very small numbers of subjects involved, little weight can be attached to this finding.

\section{Acknowledgments}

We wish to thank Professor John Hinton and Dr Malcolm
Lader for their helpful advice, Mr Desmond Macdonald, senior technician and members of the nursing staff for their $\bar{D}$ assistance, and the subjects for their patient co-operation.

\section{References}

Christie, M.J. \& Venables, P.H. (1971a) Characteristics of palmar skin potential and conductance in relaxed human $\overparen{\Phi}$ subjects. Psychophysiology, 8, 525.

Christie, M.J. \& Venables, P.H. (1971b) Effects on 'basal' skin potential level of varying the concentration of an external electrolyte. Journal of Psychosomatic Research, 15, $\vec{\circ}$ 343.

Christie, M.J. \& Venables, P.H. (1971c) Basal palmar skin $\vec{\omega}$ potential and the electrocardiogram T-wave. Psycho- $\frac{\sigma}{\partial}$ physiology, 8, 779.

Dillon, R.F. \& WeBB, W.B. (1965) Threshold of arousal from 'activated' sleep in the rat. Journal of Comparative of and Physiological Psychology, 59, 446.

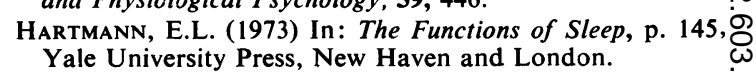

Johnson, L.C. (1963) Some attributes of spontaneous autonomic activity. Journal of Comparative and Physiolo-o gical Psychology, 56, 415.

Johnson, L.C. \& Lubin, A. (1966) Spontaneous electro-dermal activity during waking and sleeping. Psycho- ్ֶָ physiology, 3, 8 .

Koumans, A.J.R., TURSKy, B. \& Solomon, P. (1968) Electrodermal levels and fluctuations during normal sleep. Psychophysiology, 5, 300.

LADER, M.H. \& MONTAGU, J.D. (1962) The psycho-galva बै reflex: a pharmocological study of the peripheral mect:anism. Journal of Neurology, Neurosurgery and Psychiat
25, 126 .

LADER, M.H. \& WING, L. (1964) Habituation of the psychogalvanic reflex in patients with anxiety states and in normal subjects. Journal of Neurology, Neurosurgery and Psychia- $\frac{0}{1}$ try, 27, 210.

LADER, M.H. \& WiNG, L. (1965) Comparative bioassay of $\overrightarrow{\vec{\theta}}$ chlordiazepoxide and amylobarbitone sodium therapies in $\frac{0}{3}$ patients with anxiety states using physiological and clinical measures. Journal of Neurology, Neurosurgery and Psychiatry, 28, 414.

LADER, M.H. \& Wing, L. (1969) Physiological measures ino agitated and retarded depressed patients. Journal of 3 Psychiatric Research, 7, 89.

Leiderman, P.H. \& Shapiro, D. (1964) Studies on the galvanic skin potential level: some behavioural correlates. Journal of Psychosomatic Research, 7, 277.

MCCarley, R.W. \& Hobson, J. (1971) Single neuron activity in cat giganto-cellular tegmental field: selectivity of을 discharge in desynchronized sleep. Science. Washington, 174, 1250.

Noble, P.J. \& LAder, M.H. (1971) The symptomatic correlates of the skin conductance changes in depression. $N$ Journal of Psychiatric Research, 9, 61.

Noble, P.J. \& LADER, M.H. (1972) A physiological com- N parison of 'endogenous' and 'reactive' depression. British Journal of Psychiatry, 120, 541.

O'CONELl, D.N. \& TURSKY, B. (1960) Silver-silver chloride sponge electrodes for skin potential recording. Americanto Journal of Psychology, 73, 302.

Perkins, R. \& Hinton, J. (1974) Sedative or tranquillizer? A comparison of the hypnotic effects of chlordiazepoxide and $\square$ amylobarbitone sodium. British Journal of Psychiatry, 124, $\overline{0}$ 435 . 
Rechtschaffen, A. \& Kales, A. (1968) Manual of Standardized Terminology, Techniques and Scoring System for Sleep Stages of Human Subjects. U.S. Dept of Health, Education and Welfare, Washington, D.C.

ScheffE, H. (1953) A method for judging all contrasts in the analysis of variance. Biometrica, $\mathbf{4 0 , 8 7 .}$
Shaw, D.M. \& Coppen, A. (1966) Potassium and water distribution in depression. British Journal of Psychiatry, 112, 269.

Venables, P.H. \& Martin, I. (1967) The relation of palmar sweat gland activity to level of skin potential and conductance. Psychophysiology, 3, 302. 